PROCEEDINGS OF THE

AMERICAN MATHEMATICAL SOCIETY

Volume 131, Number 5, Pages 1385-1398

S 0002-9939(02)06677-7

Article electronically published on December 6, 2002

\title{
THE WAVELET DIMENSION FUNCTION IS THE TRACE FUNCTION OF A SHIFT-INVARIANT SYSTEM
}

\author{
AMOS RON AND ZUOWEI SHEN \\ (Communicated by David R. Larson)
}

\begin{abstract}
In this note, we observe that the dimension function associated with a wavelet system is the trace of the Gramian fibers of the shift-invariant system generated by the negative dilations of the mother wavelets. When this shift-invariant system is a tight frame, each of the Gramian fibers is an orthogonal projector, and its trace, then, coincides with its rank. This connection leads to simple proofs of several results concerning the dimension function, and the arguments extend to the bi-frame case.
\end{abstract}

\section{INTRODUCTION}

Let $\Psi:=\left\{\psi_{i}\right\}_{i=1}^{r}$ be a finite subset of $L_{2}\left(\mathbb{R}^{d}\right)$. The dyadic wavelet system generated by the mother wavelets $\Psi$ is the union

$$
X(\Psi)=\bigcup_{j \in \mathbb{Z}} X_{j}(\Psi)
$$

with

$$
X_{j}(\Psi):=\mathcal{D}^{j} E(\Psi)
$$

Here, $\mathcal{D}^{j}$ is the dyadic dilation operator, i.e.,

$$
\mathcal{D}^{j} f(t):=2^{\frac{j d}{2}} f\left(2^{j} t\right),
$$

and $E(\Psi)$ is the shift-invariant (SI) system generated by $\Psi$, i.e.,

$$
E(\Psi)=\left\{E^{k} \psi: k \in \mathbb{Z}^{d}, \psi \in \Psi\right\}, \quad E^{k}: f \mapsto f(\cdot+k) .
$$

Given the set $\Psi$, our interest here is in the dimension function (Weiss' terminology) $D$ associated with $\Psi$, which is defined as follows:

$$
D(\xi):=\sum_{i=1}^{r} \sum_{k \in 2 \pi \mathbb{Z}^{d}} \sum_{j=1}^{\infty}\left|\widehat{\psi}_{i}\left(2^{j}(\xi+k)\right)\right|^{2}, \quad \xi \in \mathbb{R}^{d} .
$$

Received by the editors June 8, 2001.

2000 Mathematics Subject Classification. Primary 42C15; Secondary 42C30.

Key words and phrases. Dimension function, frames, multiresolution analysis, wavelets.

This work was supported by the US National Science Foundation under Grants DMS-9872890, DBI-9983114 and ANI-0085984, the U.S. Army Research Office under Contract DAAG55-98-10443, and the Strategic Wavelet Program Grant from the National University of Singapore. 
The dimension function was introduced in [L1] by Lemarié-Rieusset, who used it to prove that compactly supported orthonormal wavelet systems can be constructed via a multiresolution analysis (MRA). A mild smoothness assumption was assumed on the mother wavelets in this result. He then generalized the result to the biorthogonal case as well as to the case of an arbitrary integer dilation, under the assumption that the mother wavelets are compactly supported or have an exponential decay (see [L2]). Auscher in A1 and A2 extended Lemarié-Rieusset's result to the case where each of the mother wavelets has a Fourier transform which is continuous, has (at least) some mild decay at infinity, and is minimally smooth at the origin. His results in A1 and A2 cover orthonormal wavelet systems as well as biorthogonal wavelet systems in one or more dimensions. The main observation in the proofs of [L1], [L2], A1] and [A2] is that, once the dimension function is proved to assume the constant value 1, a.e., one can construct an MRA that reproduces the original wavelet system. The proof was then completed by establishing that (i) the dimension function is continuous, and (ii) the dimension function attains only integer values. Later, Gripenberg [G] and Hernández and Weiss [HW] further extended the above ideas by proving (independently) that an orthonormal wavelet system can be derived from an MRA if and only if the dimension function of its mother wavelets is equal to 1 a.e. This was then generalized to the biorthogonal case by Han [Ha] and Wang [W]. These ideas also work for general dilations in one or more dimensions (a sketch of the proof for general integer expansive dilations is found in $[\mathrm{BO}]$ ). All these results are based, either explicitly or implicitly, on the key observation of [L1], [L2], [A1] and [A2] that the values attained by the dimension function of an orthonormal wavelet system coincide with the dimension of certain subspaces of $\ell_{2}(\mathbb{Z})$. More precisely, this dimension is the rank of the range function of a certain SI space. This latter rank is referred to, by Baggett in [B], by Weber in $\mathrm{We}$ and by several other authors, as the multiplicity function.

More recently, Paluszyński, Sikić, Weiss, and Xiao [PSWX2] studied the dimension function in the context of a special type of tight wavelet frames: those that are constructed by applying Mallat's algorithm to a conjugate quadrature filter (CQF). They showed that, for this case, the dimension function may assume any nonnegative real value, and characterized the tight wavelet frames for which this function is integer-valued.

Our interest in this subject arose during the reading of the article [BRS] of Bownik, Rzeszotnik and Speegle, where we came across the definition of the dimension function. Our attempt in the present article is to show the intimate relation between this function and several elements of shift-invariant space theory [BDR2], [RS1]. The connection with wavelet systems is done, then, via the notion of quasiaffine systems, [RS2], [RS3]. We hope that the approach presented here, while similar in many of its hidden core technical details to the existing approaches, sheds new light on this subject, and helps to clarify some of the mysteries around it.

The rest of this short article is laid out as follows. In $\S 2$ and $\S 3$, we treat the case where the same wavelet system is used for decomposition and reconstruction. General wavelet systems are discussed in $\S 2$, while MRA wavelet systems are analyzed in $\S 3$. In $\S 4$, we treat the case where different wavelet systems are used for decomposition and reconstruction. 
For notational convenience, we assume that the dilation operator $\mathcal{D}$ is dyadic. However, all the results in this article extend verbatim to a general dilation based on an integer expansive dilation matrix $s$, i.e., to the dilation $\mathcal{D}: f \mapsto \sqrt{\operatorname{det} s} f(s \cdot)$.

\section{The trace FunCtion of A WAVELET SyStem}

We recall in this section some of the basic notions from the theories of wavelet frames and of shift-invariant systems. These notions include the Gramian fiberization of a shift-invariant system, the quasi-affine system, and the bracket product. We then interpret the dimension function in these terms, and draw several conclusions from that interpretation.

The Gramian fibers of a shift invariant space, and the trace function. Let $\mathrm{F}$ be a countable (or finite) subset of $L_{2}\left(\mathbb{R}^{d}\right)$, and let $E(\mathrm{~F})$ be the corresponding shift-invariant system. The SI space $\mathcal{S}(\mathrm{F})$ is defined to be the smallest closed subspace of $L_{2}\left(\mathbb{R}^{d}\right)$ that contains $E(\mathrm{~F})$. If $\{f\}$ is a singleton, we write $\mathcal{S}(f)$ instead of $\mathcal{S}(\{f\})$, and refer to $\mathcal{S}(f)$ as principal shift-invariant (PSI).

In [RS1], we analyzed the SI system $E(\mathrm{~F})$ via the inspection of the properties of two sets of "fiber operators": the Gramian fibers and the dual Gramian fibers. The Gramian fibers are a family $G(\xi), \xi \in \mathbb{R}^{d}$, with each $G(\xi)$ a nonnegative Hermitian matrix whose rows and columns are indexed by $\mathrm{F}$, and whose $(f, g)$-entry is the bracket product

$$
[\widehat{f}, \widehat{g}](\xi):=\sum_{k \in 2 \pi \mathbb{Z}^{d}} \widehat{f}(\xi+k) \widehat{g}(\xi+k) .
$$

Each operator $G(\xi)$ is considered as an endomorphism of $\ell_{2}(\mathrm{~F})$. The Gramian fibers are used in [RS1] for the characterization of many properties of the shift-invariant system $E(\mathrm{~F})$ : the Bessel property, the Riesz basis property, the frame property and more. Our focus here is on the trace $T(\xi)$ of each fiber $G(\xi)$, i.e.,

$$
T(\xi):=\sum_{f \in \mathrm{F}}[\widehat{f}, \widehat{f}](\xi) .
$$

We make the following obvious observation:

Observation 2.1. Let $X(\Psi)$ be a wavelet system. Let

$$
\mathrm{F}_{\Psi}:=\left\{2^{\frac{j d}{2}} \mathcal{D}^{j} \psi: \psi \in \Psi, j<0\right\} .
$$

Then the dimension function $D$ associated with $\Psi$ coincides with the trace function $T$ associated with the SI system $E\left(\mathrm{~F}_{\Psi}\right)$.

In view of the above observation, we refer in the sequel to the dimension function $D$ as the dimension-trace function.

Projectors. As detailed before, there are several results in the literature where the dimension-trace function is proved to be integer-valued. Our identification of this function as the trace of the Gramian does not seem, at first, to explain this phenomenon: the trace of a linear operator cannot be expected to assume, in general, an integer value. However, if $G(\xi)$ is a projector, then, trivially, its trace coincides with its rank. In this context, we recall the notion of the multiplicity function (Baggett's terminology) of a shift-invariant space

$$
M:[-\pi, \pi]^{d} \rightarrow \mathbb{Z}_{+} \quad: \quad \xi \mapsto \operatorname{rank} G(\xi) .
$$


In order to emphasize the above (i.e., the fact that the multiplicity function computes the rank of the fibers of the SI space), we refer in the sequel to this function as the multiplicity-rank function. We note that the multiplicity-rank function depends only on the underlying shift-invariant space, i.e., the multiplicity-rank function of $E(\mathrm{~F})$ coincides with that of $E\left(\mathrm{~F}^{\prime}\right)$ whenever $\mathcal{S}(\mathrm{F})=\mathcal{S}\left(\mathrm{F}^{\prime}\right)$ (cf. [BDR2; the observation goes back to Helson, $[\mathrm{H}]$ ). We also note that Baggett and his co-authors' approach of shift-invariant spaces via the tool of the projection-valued measure implicitly exploits the bracket product, hence is intimately related to the approach of [BDR2].

In view of the discussion so far, we have:

Observation 2.3. Let $E(\mathrm{~F})$ be an SI system associated with a trace function $T$. If the fiber $G(\xi)$ is a projector, then the trace function $T$ coincides at $\xi$ with the multiplicity-rank function

$$
T(\xi)=M(\xi) .
$$

Fiberization of SI tight frames. At this point, it is useful to recall the notion of a tight frame. Let $X \subset L_{2}\left(\mathbb{R}^{d}\right)$ be countable or finite and $H(X)$ be the smallest closed subspace of $L_{2}\left(\mathbb{R}^{d}\right)$ that contains $X$. Let

$$
S_{X}: L_{2}\left(\mathbb{R}^{d}\right) \rightarrow L_{2}\left(\mathbb{R}^{d}\right) \quad: \quad f \mapsto \sum_{x \in X}\langle f, x\rangle x .
$$

One says that $X$ is a Bessel system if the operator $S_{X}$ is bounded, and that $X$ is a tight frame for $H(X)$, (or tight frame, for short) if $S_{X}$ is a (-n orthogonal) projector onto $H(X)$. In particular, $X$ is a tight frame for $L_{2}\left(\mathbb{R}^{d}\right)$ whenever $S_{X}$ is the identity on $L_{2}\left(\mathbb{R}^{d}\right)$. The norm $\left\|S_{X}\right\|$ is sometimes referred to as the Bessel bound of the system $X$.

The following are some of the simpler fiberization results of [RS1]:

Result 2.5. Let $E(\mathrm{~F})$ be a shift-invariant system associated with Gramian fibers $G(\xi), \xi \in \mathbb{R}^{d}$. Then:

(i) $E(\mathrm{~F})$ is Bessel if and only if the norm function $\xi \mapsto\|G(\xi)\|=: \mathcal{G}(\xi)$ is essentially bounded. Moreover, the Bessel bound then equals $\|\mathcal{G}\|_{L_{\infty}\left(\mathbb{R}^{d}\right)}$.

(ii) $E(\mathrm{~F})$ is a tight frame for $\mathcal{S}(\mathrm{F})$ if and only if almost every fiber $G(\xi)$ is a projector.

Corollary 2.6. Let $X(\Psi)$ be a wavelet system associated with a dimension-trace function D. Let $M$ and $T$ be the multiplicity-rank and trace functions of $E\left(\mathrm{~F}_{\Psi}\right)$, respectively.

(i) Assume that the SI system $E\left(\mathrm{~F}_{\Psi}\right)$ is Bessel with Bessel bound A, then

$$
D=T \leq M A .
$$

(ii) Assume that $E\left(\mathrm{~F}_{\Psi}\right)$ is Bessel with bound 1. Then, $E\left(\mathrm{~F}_{\Psi}\right)$ is a tight frame for $\mathcal{S}\left(\mathrm{F}_{\Psi}\right)$ only if

$$
D=T=M
$$

The converse is also true, provided that $M$ is finite a.e.

Proof. For (i), since the shift invariant system $E\left(\mathrm{~F}_{\Psi}\right)$ is Bessel with Bessel bound $A$, then Result 2.5 implies that, a.e., $\mathcal{G}(\xi) \leq A$. Since, trivially, $T(\xi) \leq M(\xi) \mathcal{G}(\xi)$, we have that, a.e.,

$$
T(\xi) \leq M(\xi) \mathcal{G}(\xi) \leq M(\xi) A
$$


For (ii), assume first that $E\left(\mathrm{~F}_{\Psi}\right)$ is a tight frame for $\mathcal{S}\left(\mathrm{F}_{\Psi}\right)$. It follows from Result [2.5 (ii) that almost every fiber $G(\xi)$ is a projector. Hence, the multiplicityrank function $M$ and the trace function $T$ are the same, a.e. Invoking Observation 2.1, we obtain $D=T=M$.

Conversely, since $E\left(\mathrm{~F}_{\Psi}\right)$ is Bessel with bound 1, every eigenvalue of almost every fiber $G(\xi)$ is $\leq 1$ (by (i) of Result 2.5). This implies that, a.e., $T(\xi) \leq M(\xi)$. Now, if, at some $\xi, T(\xi)=M(\xi)$, none of the eigenvalues of $G(\xi)$ can lie in the interval $(0,1)$ (this is the place where we need the finiteness of $M(\xi)$ ) hence the spectrum of $G(\xi)$ lies in $\{0,1\}$. Thus, the self-adjoint operator $G(\xi)$ must be a projector. Invoking (ii) of Result 2.5, we conclude that $E\left(\mathrm{~F}_{\Psi}\right)$ is a tight frame.

In the sequel, given any wavelet system $X(\Psi)$, we refer to the multiplicity-rank function of $E\left(\mathrm{~F}_{\Psi}\right)$ as the multiplicity-rank function of the wavelet system $X(\Psi)$, as well. This, in general, should not create any confusion, since $X(\Psi)$ is not SI, and, hence, does not have an intrinsically-defined multiplicity-rank function.

Quasi-affine systems. So far, we have found connections between the dimension function of the wavelet system $X(\Psi)$ and certain properties of the associated shiftinvariant system $E\left(\mathrm{~F}_{\Psi}\right)$. Such observations fall short of our objective: our actual interest is in the wavelet system $X(\Psi)$, and hence we would like our assumptions to be stated directly in terms of properties of $X(\Psi)$ (and not in terms of a derived system $\left.E\left(\mathrm{~F}_{\Psi}\right)\right)$. To this end, we recall from [RS2] the notion of quasi-affine systems:

Definition. Let $X(\Psi)$ be a wavelet system. Let

$$
X_{+}(\Psi)=\bigcup_{j \geq 0} X_{j}(\Psi)
$$

be the union of the nonnegative scales in $X(\Psi)$. (Note that $X_{+}(\Psi)$ is trivially shiftinvariant.) The quasi-affine system $X^{q}(\Psi)$ associated with $X(\Psi)$ is the following shift-invariant one:

$$
X^{q}(\Psi):=E\left(\mathrm{~F}_{\Psi}\right) \cup X_{+}(\Psi) .
$$

The following is one of the basic results of [RS2]. It was used there to provide a complete characterization of all wavelet frames in terms of the fiberization of the shift-invariant $X^{q}(\Psi)$. (The notion of a "frame" is defined in (4.1).) The result is proved in [RS2] under a very mild smoothness assumption on the mother wavelets $\Psi$. That smoothness assumption was removed in CSS.

Result 2.8. Let $X(\Psi)$ be a wavelet system, and let $X^{q}(\Psi)$ be the corresponding quasi-affine system. Then:

(a) $X(\Psi)$ is a Bessel system if and only if $X^{q}(\Psi)$ is a Bessel system. The two systems have the same Bessel bound.

(b) $X(\Psi)$ is a frame for $L_{2}\left(\mathbb{R}^{d}\right)$ if and only if $X^{q}(\Psi)$ is a frame for $L_{2}\left(\mathbb{R}^{d}\right)$. The two systems also have the same upper and lower frame bounds. In particular, $X(\Psi)$ is a tight frame for $L_{2}\left(\mathbb{R}^{d}\right)$ if and only if $X^{q}(\Psi)$ is a tight frame for $L_{2}\left(\mathbb{R}^{d}\right)$.

In order to apply Result 2.8, we assume that $X(\Psi)$ is a tight frame for $L_{2}\left(\mathbb{R}^{d}\right)$. However, we still cannot invoke Corollary [2.6] because Result [2.8 only implies that the SI system $X^{q}(\Psi)=E\left(\mathrm{~F}_{\Psi}\right) \cup X_{+}(\Psi)$ is a tight frame for $L_{2}\left(\mathbb{R}^{d}\right)$. In contrast, in order to invoke Corollary [2.6] we need the component $E\left(\mathrm{~F}_{\Psi}\right)$ of $X^{q}(\Psi)$ to be 
a tight frame for $\mathcal{S}\left(\mathrm{F}_{\Psi}\right)$. To this end, we adopt the following semi-orthogonality condition. Let

$$
X_{-}(\Psi):=\bigcup_{j<0} X_{j}(\Psi) .
$$

The system $X(\Psi)$ is semi-orthogonal if the following condition holds:

$$
X_{+}(\Psi) \perp X_{-}(\Psi) .
$$

Lemma 2.9. Assume that $X(\Psi)$ is a tight frame for $L_{2}\left(\mathbb{R}^{d}\right)$. Then, the following statements are equivalent:

(i) $E\left(\mathrm{~F}_{\Psi}\right)$ is a tight frame.

(ii) $X(\Psi)$ is semi-orthogonal.

(iii) $X_{-}(\Psi)$ is a tight frame.

Proof. We prove that (i) is equivalent to (ii), the proof of the equivalence between (ii) and (iii) is similar (and simpler).

Since $X(\Psi)$ is a tight frame for $L_{2}\left(\mathbb{R}^{d}\right)$, we obtain from $\operatorname{Result} 2.8$ that $X^{q}(\Psi)$ is a tight frame for $L_{2}\left(\mathbb{R}^{d}\right)$. Now, if $X_{+}(\Psi) \perp X_{-}(\Psi)$, this semi-orthogonality condition actually implies that the shift-invariant system $X_{+}(\Psi)$ is orthogonal to the SI space $\mathcal{S}\left(X_{-}(\Psi)\right)$. The latter space contains the SI system $E\left(\mathrm{~F}_{\Psi}\right)$, and consequently the tight frame $X^{q}(\Psi)$ is the union of the mutually orthogonal sets $X_{+}(\Psi)$ and $E\left(\mathrm{~F}_{\Psi}\right)$. This trivially implies that each one of these latter sets is a tight frame.

Conversely, suppose that $X^{q}(\Psi)$ is a tight frame of $L_{2}\left(\mathbb{R}^{d}\right)$ and $E\left(\mathrm{~F}_{\Psi}\right)$ is a tight frame for $\mathcal{S}\left(\mathrm{F}_{\Psi}\right)$. Then, for an arbitrary $f \in \mathcal{S}\left(\mathrm{F}_{\Psi}\right)$ with $\|f\|=1$,

$$
1=\sum_{x \in E\left(\mathrm{~F}_{\Psi}\right)}|\langle f, x\rangle|^{2}=\sum_{x \in X^{q}(\Psi)}|\langle f, x\rangle|^{2},
$$

which implies that $\langle f, x\rangle=0$, for all $x \in X_{+}(\Psi)$. In particular, $X_{+}(\Psi) \perp X_{-}(\Psi)$.

We summarize our findings in the following theorem:

Theorem 2.10. Let $D$ and $M$ be the dimension-trace function and the multiplicityrank function of $X(\Psi)$, respectively.

(i) Assume that the wavelet system $X(\Psi)$ is Bessel with Bessel bound A. Then, a.e.,

$$
D \leq M A .
$$

(ii) Assume that $X(\Psi)$ is a tight frame for $L_{2}\left(\mathbb{R}^{d}\right)$. Consider the following conditions:

(1) $D=M$.

(2) $E\left(\mathrm{~F}_{\Psi}\right)$ is a tight frame.

(3) $X(\Psi)$ is semi-orthogonal (i.e. $X_{+}(\Psi) \perp X_{-}(\Psi)$ ).

(4) $X_{-}(\Psi)$ is a tight frame.

Then (2) $\Longleftrightarrow(3) \Longleftrightarrow(4) \Longrightarrow(1)$. The implication (1) $\Longrightarrow(2)$ is valid, also, provided that $M$ is finite a.e.

In particular, $D$ is integer-valued whenever one of the above four conditions holds.

Proof. For (i), we apply Result 2.8 to conclude that the quasi-affine system is Bessel with Bessel bound $A$, a fortiori its subsystem $E\left(\mathrm{~F}_{\Psi}\right)$ is Bessel with Bessel bound $\leq A$. The result now follows from part (i) of Corollary 2.6] 
For (ii), the equivalence of (1) and (2) follows from (ii) of Corollary 2.6] while the equivalence of (2), (3) and (4) is the statement of Lemma 2.9.

Remark. Let $X(\Psi)$ be a tight frame for $L_{2}\left(\mathbb{R}^{d}\right)$. Assume that $X_{+}(\Psi) \perp X_{-}(\Psi)$. Then, Lemma 2.9 shows that $V_{0}:=H\left(X_{-}(\Psi)\right)$ is SI, and that $E\left(\mathrm{~F}_{\Psi}\right)$ is a tight frame for this $V_{0}$. Furthermore, Theorem 2.10 shows that the space $\mathcal{S}\left(X_{-}(\Psi)\right)$ is PSI if and only if $D=1$ on its support (since an SI space is PSI if and only if its multiplicity-rank function assumes only the values 0 and 1). This provides an alternative proof to Theorem 4 of Papadakis in $[\mathrm{P}]$.

\section{The TRACE FUnCtion OF AN MRA WAVELET SYSTEM}

We analyse in this section wavelet systems that are constructed by Multiresolution Analysis (MRA, $[\mathbf{M}]$ ). For notational convenience, we restrict our attention to PSI MRAs, i.e., to the case where a single refinable function is used. The results extend to more general MRAs, as well.

Let $V_{0}$ be a PSI space and set $V_{j}:=\mathcal{D}^{j} V_{0}, j \in \mathbb{Z}$. We say that $V_{j}, j \in \mathbb{Z}$ form an MRA if (i) $V_{0}$ is refinable, i.e., $V_{0} \subset V_{1}$, and (ii) $\overline{\bigcup_{j \in \mathbb{Z}} V_{j}}=L_{2}\left(\mathbb{R}^{d}\right)$. A wavelet system $X(\Psi)$ is MRA-based ([RS2]-terminology) if $\Psi \subset V_{1}$ for some MRA. Also, if the MRA-based wavelet system $X(\Psi)$ is a frame for $L_{2}\left(\mathbb{R}^{d}\right)$, we refer to its elements as framelets.

Note that for an MRA construction, the SI system $E\left(\mathrm{~F}_{\Psi}\right)$ lies in the PSI space $V_{0}$. This implies that the multiplicity-rank function $M$ of $X(\Psi)$ is bounded by the multiplicity-rank function of $V_{0}$. Since the latter assumes only the values 0 and 1 , we conclude that $M \leq 1$. Combining this with Theorem 2.10, we obtain the following result:

Corollary 3.1. Let $X(\Psi)$ be an MRA-based wavelet system. Let $D$ be the associated dimension-trace function. Then,

(i) Assume that $X(\Psi)$ is Bessel with Bessel bound A. Then $D \leq A$ a.e..

(ii) Assume that $X(\Psi)$ is a tight framelet. Then $D \leq 1$ a.e. Furthermore, the following statements are equivalent:

(1) $D=1$ a.e. on its support.

(2) $E\left(\mathrm{~F}_{\Psi}\right)$ is a tight frame.

(3) $X(\Psi)$ is semi-orthogonal.

(4) $X_{-}(\Psi)$ is a tight frame.

Note that when $X(\Psi)$ is an orthonormal wavelet system, the semi-orthogonality condition $X_{+}(\Psi) \perp X_{-}(\Psi)$ always holds. At the same time, there are a few tight framelets $X(\Psi)$ which are not orthonormal, but still satisfy the condition $X_{+}(\Psi) \perp$ $X_{-}(\Psi)$; see e.g., the tight frames constructed by Benedetto and Li in $[\mathrm{BL}]$. For these tight framelets, the dimension-trace function assumes only the values 0 and 1 (by virtue of Corollary 3.1).

However, there are many known constructions of tight framelets that do not satisfy the semi-orthogonality condition $X_{+}(\Psi) \perp X_{-}(\Psi)$. To recall, all the constructions of tight framelets are based, either implicitly or explicitly, on one of two "construction principles": the first of which is called the Unitary Extension Principle (UEP), [ $[\mathrm{RS} 2$, while the second, more general and more recent, one is the Oblique Extension Principle (OEP), [DHRS. In all the specific novel constructions 
of compactly supported and other tight framelets (splines, box splines, pseudosplines) of [RS2], [RS4] and [DHRS], $X_{+}(\Psi)$ is never orthogonal to $X_{-}(\Psi)$ : in all these constructions, the set $X_{j}(\Psi)$ lies in closed span of the set $X_{j+1}(\Psi)$, let alone that those sets are not orthogonal to each other. Correspondingly, for all these cases, the dimension-trace function satisfies the inequalities $0 \leq D \leq 1$, but not the identity $D=1$ (on its support). Let us elaborate a little further.

Let $\phi$ be a refinable function that generates the MRA, i.e., $V_{0}:=\mathcal{S}(\phi) \subset \mathcal{D} V_{0}=$ : $V_{1}$. Then, $\widehat{\phi}(2 \cdot)=\tau_{0} \widehat{\phi}$ for some $2 \pi$-periodic function $\tau_{0}$, which is usually referred to as "the refinement mask of $\phi$ ". Moreover, since we assume that each mother wavelet lies in $V_{1}$, there exists, for each $\psi_{i} \in \Psi, i=1, \ldots, r$, a $2 \pi$-periodic function (known as the "wavelet mask") $\tau_{i}$ such that $\widehat{\psi}_{i}(2 \cdot)=\tau_{i} \widehat{\phi}$ (see [BDR1]). The vector $\left(\tau_{i}\right)_{i=0}^{r}$ of masks determines completely the wavelet system $X(\Psi)$. Hence, a major part of the theory is to establish connections between properties of the masks and properties of the corresponding wavelet system.

The particular property of $X(\Psi)$ that we study is the tight frame property. It turns out that another $2 \pi$-periodic function plays a pivotal role in that study. That function, which was introduced in [RS2], is termed there the fundamental function of the MRA. It is defined as

$$
\Theta:=\sum_{j=0}^{\infty} \sum_{i=1}^{r}\left|\tau_{i}\left(2^{j} \cdot\right)\right|^{2} \prod_{m=0}^{j-1}\left|\tau_{0}\left(2^{m} \cdot\right)\right|^{2},
$$

where $\tau_{0}, \tau_{1}, \ldots, \tau_{r}$ are the underlying masks. Then, Theorem 6.5 of [RS2] says that (assuming that $\widehat{\phi}(0) \neq 0$ ) the wavelet system $X(\Psi)$ is a tight framelet if and only if $\Theta(0)=1$ and, for every $\nu \in\{0, \pi\}^{d} \backslash 0$,

$$
\Theta(2 \cdot) \tau_{0} \overline{\tau_{0}(\cdot+\nu)}+\sum_{i=1}^{r} \tau_{i} \overline{\tau_{i}(\cdot+\nu)}=0 .
$$

Hence, in order to construct a tight framelet from a given MRA, one needs to select $\tau_{i}, i=1, \ldots, r$ and $\Theta$ that satisfy both (3.3) and (3.2). It turns out that this characterization leads to simple construction principles. For example, the UEP, in one variable and dyadic dilation, states that, in essence, the derived wavelet system $X(\Psi)$ is a tight framelet if the masks $\tau_{0}, \tau_{1}, \ldots, \tau_{r}$ satisfy a.e. the following two conditions:

$$
\sum_{i=0}^{r}\left|\tau_{i}\right|^{2}=1, \quad \sum_{i=0}^{r} \tau_{i} \overline{\tau_{i}(\cdot+\pi)}=0 .
$$

It was also shown in RS2 that $\Theta=1$ for every UEP construction. If one makes the additional assumption that $r=1$, then (3.4) simply says that the $2 \times 2$ matrix

$$
\left(\begin{array}{cc}
\tau_{0} & \tau_{0}(\cdot+\pi) \\
\tau_{1} & \tau_{1}(\cdot+\pi)
\end{array}\right)
$$

is unitary. Thus, the construction of tight framelets with a single mother wavelet by the UEP from an MRA that is generated by a univariate dyadic refinable function $\phi$ can succeed only when the refinement mask $\tau_{0}$ of $\phi$ satisfies the condition $\left|\tau_{0}\right|^{2}+$ $\left|\tau_{0}(\cdot+\pi)\right|^{2}=1$ a.e. (a mask that satisfies this condition is known as a conjugate quadrature filter $(=: \mathrm{CQF})$ ). We note that the UEP implies, thus, that a $C Q F$ construction (i.e., an application of Mallat's algorithm, [M], to a CQF mask) yields a tight framelet. Lawton, in $[\mathrm{La}$, was the first to prove the latter fact (for finitely 
supported filters), long before RS2 obtained this result (for general filters) by specializing the UEP. We refer to $\S 6$ of [RS2] for more details, and in particular to Theorem 6.5 and Corollary 6.7 there.

The dimension-trace function enters the discussion due to the following identity, whose verification involves straightforward computations (such as those used in the proof of Lemma 6.2 of [RS2]):

$$
D=\Theta[\widehat{\phi}, \widehat{\phi}] .
$$

This shows that the fundamental function $\Theta$ and the underlying refinable function $\phi$ of the MRA completely determine the dimension-trace function of the corresponding framelet system $X(\Psi)$. In particular, we have:

Corollary 3.6. Let $X(\Psi)$ be a framelet that is constructed via the UEP from an MRA generated by a refinable function $\phi$. Then, $D=[\widehat{\phi}, \widehat{\phi}]$. In particular, if $\phi$ is compactly supported, then $D$ is a trigonometric polynomial.

Proof. Since $X(\Psi)$ is constructed via the UEP, the function $\Theta$ must equal 1 on its support (by Corollary 6.7 of [RS2]). Hence, $D=[\widehat{\phi}, \widehat{\phi}]$ by (3.5). If $\phi$ is compactly supported, its autocorrelation function $[\widehat{\phi}, \widehat{\phi}]$ is a trigonometric polynomial.

There are various UEP constructions of smooth compactly supported tight framelet systems for which $[\widehat{\phi}, \widehat{\phi}]$ vanishes at some points (see, e.g., the bivariate boxlets that are derived in [RS4] from the 4-directional box spline $\phi$ ). Hence, Corollary 3.6 shows that the corresponding dimension-trace function in each of these examples is a trigonometric polynomial that assumes all values between 0 and 1.

Remark. The CQF construction was extensively studied recently by Paluszyński, Sikić, Weiss and Xiao in [PSWX1] and [PSWX2], where a deeper understanding of conjugate quadrature filters was obtained. In particular, Corollary 3.6 and part of Corollary [3.1] were proven, for CQF constructions, in Theorem 3.18 and Proposition 3.12 of PSWX2].

We conclude this section with the following final remark:

Remark. Is the dimension-trace function a useful tool for general tight framelets? We have shown above that one should not expect the dimension-trace function of a general tight framelet to coincide with its multiplicity-rank function. There are other useful properties of a tight framelet that are always encoded in the dimension function. For example, the approximation order of the tight framelet system, [DHRS], is characterized by the order of the zero of the function $1-\Theta[\widehat{\phi}, \widehat{\phi}]$ at the origin. Of course, in view of (3.5), such results can be rewritten in terms of the dimension-trace function $D$.

\section{The MiXed TRACE FUnCtion OF DUAL SYSTEMS}

Our focus so far was primarily on the case of tight frames (including orthonormal systems). The results can be easily modified to cover the case of bi-frames. We forgo generalizing all the tight frame results, and concentrate, instead, on the bi-frame counterpart of Theorem 2.10 . 
We start with some necessary definitions. A Bessel system $X$ is a frame for $H(X)$, the closed span of $X$, if the operator $S_{X}$ (cf. (2.4) has a closed range. An alternative definition is that

$$
C_{1}\|f\|_{L_{2}\left(\mathbb{R}^{d}\right)}^{2} \leq\left\|S_{X} f\right\|_{L_{2}\left(\mathbb{R}^{d}\right)}^{2} \leq C_{2}\|f\|_{L_{2}\left(\mathbb{R}^{d}\right)}^{2},
$$

for every $f$ in $H(X)$ and for some $f$-independent positive constants $C_{1}$ and $C_{2}$.

Now, let $\mathrm{R}: X \rightarrow L_{2}\left(\mathbb{R}^{d}\right)$ be some assignment, and assume that both $X$ and $\mathrm{R}(X)$ are Bessel systems. Let

$$
S f:=\sum_{x \in X}\langle f, \mathrm{R} x\rangle x, \quad f \in L_{2}\left(\mathbb{R}^{d}\right) .
$$

If the perfect reconstruction property $S f=f$ holds for every $f \in H(X)$, then $X$ is a frame for $H(X)$, and $\mathrm{R} X$ is referred to as a dual system of $X$. The (ordered) pair $(X, \mathrm{R} X)$ is then called a representation pair. If, in addition, $\operatorname{ker} S$ coincides with the orthogonal complement of $H(\mathrm{R} X)$, then $\mathrm{R} X$ is also a frame (for $H(\mathrm{R} X)$ ), and the pair $(\mathrm{R} X, X)$ becomes a representation system, too. In this case, we refer to the (unordered) pair $(X, \mathrm{R} X)$ as a bi-frame. We note that a representation system $(X, \mathrm{R} X)$ is automatically a bi-frame, whenever $H(X)=H(\mathrm{R} X)$. As one expects, it is far easier to answer questions of the form "is $(X, \mathrm{R} X)$ a bi-frame?", as compared to the question "is $X$ a frame?". To a large degree, the analysis of the bi-frame property of $(X, \mathrm{R} X)$ is parallel to the analysis of tight frames, while the intrinsic analysis of a frame $X$ poses a much greater challenge.

Let $(E(\mathrm{~F}), E(\mathrm{RF}))$ be two SI systems. (Note that, formally, the map $\mathrm{R}$ is defined only on $\mathrm{F}$, but, its extension to a map on $E(\mathrm{~F})$ should be self-understood.) The mixed Gramian fiber $G(\xi), \xi \in \mathbb{R}^{d}$, associated with these systems is a matrix whose rows and columns are indexed by $\mathrm{F}$, and whose $(f, g)$ entry is $[\widehat{g}, \widehat{\mathrm{Rf}}](\xi)$. Then, the fiberization analysis of RS1 can be applied to conclude that if $(E(\mathrm{~F}), E(\mathrm{RF}))$ is a representation pair, then almost every fiber $G(\xi)$ is a projector.

Let $X(\Psi)$ and $X(\mathrm{R} \Psi)$ be two wavelet Bessel systems (corresponding to the same dilation operator, which, as before, we assume to be dyadic). Let $E\left(\mathrm{~F}_{\Psi}\right)$ and $E\left(\mathrm{~F}_{\mathrm{R} \Psi}\right)$ be the two SI systems associated with $X(\Psi)$ and $X(\mathrm{R} \Psi)$ as given in $\S 2$. Denote by $G_{\Psi, \mathrm{R} \Psi}(\xi), \xi \in \mathbb{R}^{d}$ the mixed Gramian fiber operators of $\left(E\left(\mathrm{~F}_{\Psi}\right), E\left(\mathrm{~F}_{\mathrm{R} \Psi}\right)\right)$. The mixed rank function of $(X(\Psi), X(\mathrm{R} \Psi))$ is

$$
M_{\Psi, \mathrm{R} \Psi}:[-\pi, \pi]^{d} \rightarrow \mathbb{Z}_{+} \quad: \quad \xi \mapsto \operatorname{rank} G_{\Psi, \mathrm{R} \Psi}(\xi) .
$$

Let $D_{\Psi}$ and $D_{\mathrm{R} \Psi}$ be the dimension-trace functions of the wavelet systems $X(\Psi)$ and $X(\mathrm{R} \Psi)$ respectively. The mixed trace function $D_{\Psi, \mathrm{R} \Psi}(\xi)$ of $(X(\Psi), X(\mathrm{R} \Psi))$ is defined, at a point $\xi \in \mathbb{R}^{d}$ that satisfies $\max \left\{D_{\Psi}(\xi), D_{\mathrm{R} \Psi}(\xi)\right\}<\infty$, as

$$
D_{\Psi, \mathrm{R} \Psi}(\xi):=\sum_{\psi \in \Psi} \sum_{j>0}\left[\widehat{\psi}\left(2^{j} \cdot\right), \widehat{\mathrm{R} \psi}\left(2^{j} \cdot\right)\right](\xi) .
$$

Since $\max \left\{D_{\Psi}(\xi), D_{\mathrm{R} \Psi}(\xi)\right\}<\infty$, the sum that defines $D_{\Psi, \mathrm{R} \Psi}(\xi)$ converges absolutely, hence can be used to define the trace of the operator $G_{\Psi, \mathrm{R} \Psi}(\xi)$. Furthermore, the following observation follows from the basic facts on trace-class operators (see e.g. Appendix 2 of $[\mathrm{F}]$ ).

Observation 4.3. Let $X(\Psi)$ and $X(\mathrm{R} \Psi)$ be two given wavelet systems. Assume that $\max \left\{D_{\Psi}(\xi), D_{\mathrm{R} \Psi}(\xi)\right\}<\infty$ and assume that $G_{\Psi, \mathrm{R} \Psi}(\xi)$ is a projector, then

$$
D_{\Psi, \mathrm{R} \Psi}(\xi)=M_{\Psi, \mathrm{R} \Psi}(\xi) .
$$


The following three results are analogous to Theorem 2.10 These results require a very mild smoothness condition on the wavelet systems $X(\Psi)$ and $X(\mathrm{R} \Psi)$ (viz. condition (4.6) of [RS2]; that condition is used in the proof of Theorem 1 of [RS3], and the latter is invoked in the proof below). The condition is so mild (even the Haar system, in all dimensions, satisfies it), that we forgo mentioning it in the formal statement.

Proposition 4.4. Let $(X(\Psi), X(\mathrm{R} \Psi))$ be a pair of bi-frames for $L_{2}\left(\mathbb{R}^{d}\right)$. Assume that the following semi-bi-orthogonality condition holds:

$$
X_{-}(\Psi) \perp X_{+}(\mathrm{R} \Psi) .
$$

Then the equality

$$
D_{\Psi, \mathrm{R} \Psi}=M_{\Psi, \mathrm{R} \Psi}
$$

holds at the points $\xi \in \mathbb{R}^{d}$ where $\max \left\{D_{\Psi}(\xi), D_{\mathrm{R} \Psi}(\xi)\right\}<\infty$. In particular, $D_{\Psi, \mathrm{R} \Psi}$ assumes integer values at those points.

Proof. Since $(X(\Psi), X(\mathrm{R} \Psi))$ is a pair of bi-frames, their quasi-affine counterparts $\left(X^{q}(\Psi), X^{q}(\mathrm{R} \Psi)\right)$ is a pair of bi-frames by Theorem 1 of [RS3]. Condition (4.5) implies that $E\left(\mathrm{~F}_{\Psi}\right)$ is orthogonal to $X_{+}(\mathrm{R} \Psi)$. This, together with the fact that $\left(X^{q}(\Psi), X^{q}(\mathrm{R} \Psi)\right)$ is a bi-frame, implies that $\left(E\left(\mathrm{~F}_{\Psi}\right), E\left(\mathrm{~F}_{\mathrm{R} \Psi}\right)\right)$ is a representation pair. Hence, almost every fiber $G_{\Psi, \mathrm{R} \Psi}(\xi), \xi \in \mathbb{R}^{d}$ is a projector. It follows from Observation 4.3 that $D_{\Psi, \mathrm{R} \Psi}(\xi)=M_{\Psi, \mathrm{R} \Psi}(\xi)$ whenever $\max \left\{D_{\Psi}(\xi), D_{\mathrm{R} \Psi}(\xi)\right\}<$ $\infty$.

Next, we will attempt to establish a converse to the above proposition, i.e., we will show that (under some additional assumptions) condition (4.6) on the dimension-trace function implies the semi-bi-orthogonality condition (4.5) (compare with part (ii) of Theorem 2.10).

First we need the following lemma.

Lemma 4.7. Let $A$ be a bounded linear operator from a Hilbert space $H$ to another Hilbert space $H^{\prime}$. Let $B: H^{\prime} \rightarrow H$ be bounded and linear, too. If $A B$ is a projector, and, if $A$ is injective on $\operatorname{ran} B$, then $B A$ is the identity on $\operatorname{ran} B$.

Proof. By assumption, $A B A B=A B$. Since $A$ is injective on $\operatorname{ran} B$, it follows that $B A B=B$. Thus, $B A$, indeed, is the identity on $\operatorname{ran} B$.

Theorem 4.8. Let $(X(\Psi), X(\mathrm{R} \Psi))$ be a bi-frame for $L_{2}\left(\mathbb{R}^{d}\right)$. Assume that, a.e., $\max \left\{D_{\Psi}, D_{\mathrm{R} \Psi}\right\}<\infty$ Then, the semi-bi-orthogonality conditions

$$
X_{-}(\Psi) \perp X_{+}(\mathrm{R} \Psi) \quad \text { and } \quad X_{-}(\mathrm{R} \Psi) \perp X_{+}(\Psi)
$$

are equivalent to the following three conditions:

$$
\begin{gathered}
D_{\Psi, \mathrm{R} \Psi}=M_{\Psi, \mathrm{R} \Psi} \quad \text { a.e., } \\
S\left(F_{\Psi}\right) \cap S\left(F_{\mathrm{R} \Psi}\right)^{\perp}=0,
\end{gathered}
$$

and

$$
\langle x, \mathrm{R} y\rangle=\langle\mathrm{R} x, y\rangle, \quad \forall x \in X_{-}(\Psi), \quad y \in X_{+}(\Psi) .
$$

Proof. The fact that the condition $X_{-}(\Psi) \perp X_{+}(\mathrm{R} \Psi)$ implies (4.10) was proved in Proposition 4.4. Since 0 is the only element orthogonal to all the elements of $X(\mathrm{R} \Psi)$, (4.11) follows from the first condition in (4.9). It is clear that (4.9) implies 
that $\langle x, \mathrm{R} y\rangle=0=\langle\mathrm{R} x, y\rangle$ for all $x \in X_{-}(\Psi)$ and $y \in X_{+}(\Psi)$, and, therefore, (4.12) is valid.

We now prove the converse implication. First, let us note that, since the wavelet system pair $(X(\Psi), X(\mathrm{R} \Psi))$ is a bi-frame for $L_{2}\left(\mathbb{R}^{d}\right)$, Theorem 1 of [RS3] implies that the quasi-affine pair $\left(X^{q}(\Psi), X^{q}(\mathrm{R} \Psi)\right)$ is a bi-frame for $L_{2}\left(\mathbb{R}^{d}\right)$, too.

Now, as we explained previously, equality (4.10) implies that almost every fiber of the mixed Gramian $G_{\Psi, \mathrm{R} \Psi}$ is a projector. The fiberization techniques in $\S 4$ of [RS1] then apply to show that the operator $A B$ is a projector, with $A$ the analysis map

$$
A: L_{2}\left(\mathbb{R}^{d}\right) \rightarrow \ell_{2}(X): f \mapsto(\langle f, \mathrm{R} x\rangle)_{x \in X}
$$

and $B$ is the synthesis map

$$
B: \ell_{2}(X) \rightarrow L_{2}\left(\mathbb{R}^{d}\right): c \mapsto \sum_{x \in X} c(x) x
$$

and where

$$
X:=E\left(\mathrm{~F}_{\Psi}\right)
$$

Assumption (4.11) exactly says that $A$ does not vanish on the range of $B$, and thus we can invoke Lemma 4.7 to conclude that $B A$ is the identity on the range of $B$, i.e., on the space $S\left(\mathrm{~F}_{\Psi}\right)$. Writing $B A$ explicitly, one sees that we have just proved that the pair $\left(E\left(\mathrm{~F}_{\Psi}\right), E\left(\mathrm{~F}_{\mathrm{R} \psi}\right)\right)$ is a representation pair for $S\left(\mathrm{~F}_{\Psi}\right)$.

We can now complete the proof by following the argument in the proof of Lemma 2.9; let $y \in X_{-}(\Psi)$. Then, with $S$ as in (4.2), we have, for the choice $X:=$ $X^{q}(\Psi)$ there, that $S y=y$ (since $\left(X^{q}(\Psi), X^{q}(\mathrm{R} \Psi)\right)$ is a bi-frame for $\left.L_{2}\left(\mathbb{R}^{d}\right)\right)$. Thus, invoking (4.12), we have

$$
\begin{aligned}
\langle y, \mathrm{R} y\rangle & =\langle S y, \mathrm{R} y\rangle=\sum_{x \in X^{q}(\Psi)}\langle y, \mathrm{R} x\rangle\langle x, \mathrm{R} y\rangle \\
& =\sum_{x \in E\left(\mathrm{~F}_{\Psi}\right)}\langle y, \mathrm{R} x\rangle\langle x, \mathrm{R} y\rangle+\sum_{x \in X_{+}(\Psi)}|\langle y, \mathrm{R} x\rangle|^{2} .
\end{aligned}
$$

On the other hand, if we choose $X:=E\left(F_{\Psi}\right)$ in (4.2), we still have that $S y=y$ (since we proved before that $\left(E\left(\mathrm{~F}_{\Psi}\right), E\left(\mathrm{~F}_{\mathrm{R} \Psi}\right)\right)$ is a representation pair), and hence by the same argument

$$
\langle y, \mathrm{R} y\rangle=\sum_{x \in E\left(\mathrm{~F}_{\Psi}\right)}\langle y, \mathrm{R} x\rangle\langle x, \mathrm{R} y\rangle .
$$

Hence, we conclude that $y \perp X_{+}(\mathrm{R} \Psi)$ for all $y \in X_{-}(\Psi)$, i.e. $X_{-}(\Psi) \perp X_{+}(\mathrm{R} \Psi)$. This, together with (4.12), also implies $X_{-}(\mathrm{R} \Psi) \perp X_{+}(\Psi)$.

We note that the above result covers the case of a minimal bi-frame. Recall that a bi-frame $(X, \mathrm{R} X)$ is minimal if the projector $A B$ (with $A, B$ as in the proof of the last theorem) is self-adjoint. In this case, one can prove that the map $\mathrm{R}$ is the restriction to $X$ of the operator $\left(S_{X}\right)^{-1}$ (cf. (2.4)). We find it convenient to extend $\mathrm{R}$ in this case, i.e., to define $\mathrm{R}:=\left(S_{X}\right)^{-1}$. We also note that the notion of minimality is symmetric, i.e., given a bi-frame $(X, \mathrm{R} X), \mathrm{R} X$ is the minimal dual frame of $X$ if and only if $X$ is the minimal dual system of $\mathrm{R} X$.

Corollary 4.13. Let $(X(\Psi), X(\mathrm{R} \Psi))$ be a minimal wavelet bi-frame. Assume that, a.e., $\max \left\{D_{\Psi}, D_{\mathrm{R} \Psi}\right\}<\infty$. Then the following three conditions are equivalent:

(1) $D_{\Psi, \mathrm{R} \Psi}=M_{\Psi, \mathrm{R} \Psi}$ a.e. 
(2) $X_{-}(\Psi) \perp X_{+}(\mathrm{R} \Psi)$.

(3) $X_{-}(\mathrm{R} \Psi) \perp X_{+}(\Psi)$.

Proof. The equivalence between (2) and (3) is immediate, since, thanks to the minimality, R can be identified with the self-adjoint operator $S_{X(\Psi)}^{-1}$.

The equivalence between (2) and (1) will follow from Theorem 4.8, once we verify that conditions (4.11) and (4.12) hold, whenever $(X(\Psi), X(\mathrm{R} \Psi))$ is a minimal wavelet bi-frame. Condition (4.12) follows, indeed, from the self-adjointness of R. As for condition (4.11), we first invoke Theorem 1 of [RS3] to conclude that the quasi-affine pair $\left(X^{q}(\Psi), X^{q}(\mathrm{R} \Psi)\right)$ is a minimal bi-frame. Using the positivity of $\mathrm{R}:=S_{X^{q}(\Psi)}^{-1}$, it is easy to verify that (4.11) holds (based on the fact that $E\left(\mathrm{~F}_{\Psi}\right)$ is a subset of $\left.X^{q}(\Psi)\right)$.

\section{REFERENCES}

[A1] Auscher, P., Toute base d'ondelettes régulières de $L^{2}(\mathbb{R})$ : Analyse Multi-Résolution régulière, CRAS Série 315, 1, 1992, 1227-1230. MR 94e:42041

[A2] Auscher, P., Solution of two problems on wavelets, J. Geom. Anal., 5, 1995, 181-236. MR 96g:42016

[B] Baggett, L. W., An abstract interpretation of wavelet dimension function using group representation, J. Funct. Anal., 173, 2000, 1-20. MR 2001j:42028

[BL] Benedetto, J., Li, S. D., The theory of multiresolution analysis frames and applications to filter banks, Appl. Comput. Harmonic Anal., 5(4), 1998, 389-427. MR 99k:42054

[BDR1] de Boor, C., DeVore, R., Ron, A., Approximation from shift-invariant subspaces of $L_{2}\left(\mathbb{R}^{d}\right)$, Trans. Amer. Math. Soc., 341, 1994, 787-806. MR 94d:41028

[BDR2] de Boor, C., DeVore, R., Ron, A., The structure of finitely generated shift-invariant spaces in $L_{2}\left(\mathbb{R}^{d}\right)$, J. Funct. Anal., 119(1), 1994, 37-78. MR 95g:46050

[Bo] J Bownik, M., The structure of shift invariant subspaces of $L^{2}\left(\mathbb{R}^{n}\right)$, J. Funct. Anal., 177, 2000, 282-309. MR 2001k:42037

[BRS] J Bownik, M., Rzeszotnik, Z., Speegle, D., A characterization of dimension function of wavelets, Appl. Comput. Harmonic Anal., 10, 2001, 71-92. MR 2001m:42058

[CSS] Chui, C. K., Shi, X., Stockler, J., Affine frames, quasi-frames and there duals, Appl. Comput. Harmonic Anal., 8, 1998, 1-17. MR 99b:42037

[DHRS] Daubechies, I., Han, B., Ron, A., Shen, Z., Framelets: MRA-based constructions of wavelet frames, preprint, 2001, Ftp site: ftp://ftp.cs.wisc.edu/Approx file dhrs.ps.

[F] B. Folland, G. B., A Course in Abstract Harmonic Analysis, CRC Press (Boca Raton), 1995. MR 98c: 43001

[G] Gripenberg, G., A necessary and sufficient condition for the existence of father wavelet, Studia Math., 114, 1995, 207-226. MR 96d:42049

[Ha] Han, Bin, Some applications of projection operators in wavelets, Acta Math. Sinica, New Series, 11, 1995, 105-112. MR 97k:42061

$[\mathrm{H}] \quad$ Helson, H., Lectures on Invariant Subspace, Academic Press (New York), 1964. MR 30:1409

[HW] Hernández, E., Weiss, G., A first Course on Wavelets, Studies in Advanced Mathematics, CRC Press (Boca Raton FL), 1996. MR 97i:42015

[La] Lawton, W., Tight frames of compactly supported affine wavelets, J. Math. Phys., 31, 1990, 1898-1901. MR 92a:81068

[L1] Lemarié-Rieusset, P. G., Existence de "fonction-pére"pour les ondelettes á support compact, C.R. Acad. Sci. Paris, Ser. I., Math., 314, 1992, 17-19. MR 93c:42033

[L2] Lemarié-Rieusset, P. G., Ondelettes généralisées et fonctions d'échelle á support compact, Rev. Mat. Iberoamericana, 9, 1993, 333-371. MR 94i:42045

[M] Mallat, S., Multiresolution approximations and wavelet orthonormal bases of $L^{2}(\mathbb{R})$, Trans. Amer. Math. Soc., 315, 1989, 69-87. MR 90e:42046

[PSWX1] Paluszyński, M., Sikić, H., Weiss, G., Xiao, X., Generalized low pass filters and MRA frame wavelets, J. Geom. Anal., 11, 2001, 311-342.

[PSWX2] Paluszyński, M., Sikić, H., Weiss, G., Xiao, X., Tight frame wavelets, their dimension functions, MRA tight frame wavelets and connectivity properties, preprint, 2001. 
[P] Papadakis, M., On the dimension function of orthonormal wavelets, Proc. Amer. Math. Soc., 128, 1999, 2042-2049. MR 2000m:42031

[RS1] Ron, A., Shen, Z., Frames and stable bases for shift-invariant subspaces of $L_{2}\left(\mathbb{R}^{d}\right)$, Canad. J. Math., 47(5), 1995, 1051-1094; Ftp site: ftp://ftp.cs.wisc.edu/Approx file frame1.ps. MR 96k: 42049

[RS2] Ron, A., Shen, Z., Affine systems in $L_{2}\left(\mathbb{R}^{d}\right)$ : the analysis of the analysis operator, J. Funct. Anal., 148, 1997, 408-447; Ftp site: ftp://ftp.cs.wisc.edu/Approx file affine.ps. MR 99g:42043

[RS3] Ron, A., Shen, Z., Affine systems in $L_{2}\left(\mathbb{R}^{d}\right)$ II: dual systems, J. Fourier Anal. Appl., 3, 1997, 617-637; Ftp site: ftp://ftp.cs.wisc.edu/Approx file dframe.ps. MR 99g:42044

[RS4] Ron, A., Shen, Z., Compactly supported tight affine spline frames in $L_{2}\left(\mathbb{R}^{d}\right)$, Math. Comp., 67, 1998, 191-207; Ftp site: ftp://ftp.cs.wisc.edu/Approx file tight.ps. MR 98c: 42035

[W] Wang, X., The study of wavelets from properties of their Fourier transforms, Ph.D. Thesis, Washington University, St. Louis, MO, 1995.

[We] Weber, E., Applications of the wavelet multiplicity function, Contemp. Math., 247, 1999, 297-306. MR 2001f:42064

Computer Sciences Department, University of Wisconsin-Madison, 1210 West DayTON, MADISON, WISCONSIN 53706

E-mail address: amos@cs.wisc.edu

Department of Mathematics, National University of Singapore, 10 Kent Ridge CresCENT, SingaPORE 119260

E-mail address: matzuows@leonis.nus.edu.sg 